\title{
Desenvolvendo uma plataforma com Gêmeo Digital baseada na web para análise de braço robótico
}

\author{
Daniel Fernandes G. de Oliveira, Rummenigge Rudson Dantas \\ Escola de Ciências e Tecnologia - Universidade Federal do Rio Grande \\ do Norte (UFRN) - Natal, RN - Brazil \\ danielfeenandes@gmail.com,rudsondant@gmail.com
}

\begin{abstract}
Resumo - Este artigo descreve a criação e os resultados de simulação de um sistema para criar gêmeos digitais baseados na web. $O$ sistema possui um simulador visual 3D que roda diretamente no navegador, suportando visualização de dados com painel e realidade aumentada. Para validar o sistema, um braço físico robótico foi construído com o LEGO, no qual foram colocados sensores conectados a um microcontrolador, o qual envia os dados para um banco de dados na web. Sua contraparte digital foi criada em nosso simulador.
\end{abstract}

\begin{abstract}
This article describes the creation of a system for creating web-based digital twins. The system has a 3D visual simulator, and this simulator runs directly in the browser. Supports panel data visualization and augmented reality. To validate the system, a robotic physical arm was built with LEGO. In this arm were placed sensors connected to a microcontroller. The microcontroller sends the data to a database on the web. Your digital counterpart was created in our simulator. The article presents the results of this simulation.
\end{abstract}

\section{INTRODUÇão}

O processo de fabricação industrial evoluiu ao longo dos anos e o conjunto de condições que caracterizam essa evolução é chamado de revolução industrial. Três dessas revoluções já foram notadas ao longo da história. Acredita-se que o mundo está atualmente se movendo em direção à $4^{\mathrm{a}}$ revolução, que está ligada à criação de "objetos inteligentes", cuja capacidade é aplicar sensores ao meio ambiente e nos enviar os dados de seus 
sensores [1]. Essa comunicação de objetos inteligentes conosco ocorre por meio da comunicação sem fio, seja pela Internet ou por um transmissor de curto alcance, como antenas Bluetooth. Se vários desses objetos têm uma conexão sem fio com a Internet, eles podem trocar dados entre si formando a Internet das Coisas [2].

A capacidade de trocar dados entre coisas que operam em uma linha de montagem de fábrica permitiria que o equipamento, agora inteligente, colaborasse no processo de fabricação ao invés de serem apenas agentes passivos. Esta colaboração de máquinas no processo de fabricação seria tão inovador que foi considerado o nascimento da chamada indústria 4.0 [3].

Essa nova indústria não é determinada apenas pela presença de Sistemas de Internet das Coisas no processo industrial. Lasi et al. [4] argumenta que também envolve mudanças na produção, com a introdução de um sistema de produção flexível, que para Weyer et al. [5] ocorre através da implantação de sistemas ciber-físicos [6], definidos como sistemas elétrico mls e / ou mecânicos com maior poder computacional. A criação de sistemas ciberfísicos ocorre com a adição de sistemas embarcados [7] em equipamentos elétricos e/ou mecânicos, que anteriormente não possuíam poder de processamento e armazenamento interno de dados [8].

Segundo Weyer et al. [14], a estrutura da indústria 4.0 envolve dois outros aspectos além da implantação de sistemas ciberfísicos, o Produto Inteligente e o Operador Aumentado. O primeiro é baseado na descrição do produto por meio de arquivos de metadados, que armazenam de forma eficiente muitas informações estratégicas sobre o produto [18]. O Operador Aumentado envolve o uso de tecnologias que permitem que os operadores da fábrica visualizem dados do processo de fabricação rápida e dinamicamente para tomar decisões. As interfaces que permitem essa visualização são construídas com Realidade Aumentada (RA) [20].

Entre essas tecnologias que o advento da Indústria 4.0 oferece às indústrias está o uso de gêmeos digitais. Um gêmeo digital é uma representação digital (ou em outras palavras, virtual) de um objeto do mundo real [1]. Esta representação digital é feita pela combinação de tecnologias de sensoriamento, internet de coisas [2], análise de dados e ambientes virtuais de simulação.

Um objeto que tenha sensores conectados à internet pode enviar dados selecionados sobre esse objeto em tempo real. Esses dados vão para um servidor na nuvem e lá ficam armazenados em um banco de dados e podem ser usados para montar um histórico do comportamento do objeto, com propósitos muito diferentes, como visualização posterior, inspeções ou até mesmo o desenvolvimento de modelos de previsão para simular o comportamento do objeto sob circunstâncias mais gerais. Esse conjunto de ações representa o funcionamento de um gêmeo digital, que pode ser visto como uma visualização sincronizada dos dados de um objeto [3].

Essa definição de gêmeo digital foi proposta por Grieves [4], que discute o uso do Product Life Cycle Management (PLM) [5],para quem a motivação para criar um gêmeo digital é reduzir os custos de produção de um novo produto [6]. Além disso, outras etapas do PLM, como a manutenção do produto, também podem ser otimizadas pelo uso de gêmeos digitais [7]. O conceito de gêmeo digital é recente, mas já existem alguns sistemas para a construção desses produtos disponíveis no mercado em plataformas listadas por Qi 
et al. [32] , porém, todas as soluções apresentadas são proprietárias. Nossa proposta neste trabalho é apresentar uma solução que utiliza software gratuito e baseado na web, chamado DTEAM (Digital Twin Environment for Augmented Management), cuja plataforma utiliza RA para visualização de dados.

Para apresentar o DTEAM, introduziremos, Na seção 2, um histórico da área com a listagem de alguns trabalhos relacionados. Na seção 3 descrevemos o nosso sistema, cujos resultados serão discutidos em seguida, na seção 4. Por fim, na seção 5, apresentamos a conclusão do trabalho

\section{Trabalhos Relacionados}

A tecnologia dos gêmeos digitais foi empregada primeiramente no projeto e fabricação de aeronaves, quando A NASA e a Força Aérea Americana usaram para fabricar veículos novos [8]. Tuegel et al [9] descreve o uso de gêmeos digitais como uma forma de prever o desempenho de uma aeronave durante sua vida útil. Luo et al. [25] desenvolveram um sistema para criar gêmeos digitais para máquinas $\mathrm{CNC}$ em um trabalho que apresenta o sistema organizado em 3 camadas: camada física, camada digital e modelo de dados. Esse modelo de arquitetura de 3 camadas serve como base para nosso trabalho e sua terminologia também é usada por outros trabalhos que serão citados nesta seção. A parte física do gêmeo tem sensores que coletam dados. Os dados coletados só serão úteis quando visualizados e, por isso, é possível encontrar diferentes abordagens para visualização, mas o uso de Dashboards [32] e Augmented Reality [19] são os mais citados. Considerados os fundamentos de um sistema para gêmeos digitais, podemos abordar alguns trabalhos na área.

\section{A. $\quad$ Sistemas de Gêmeos Digitais}

Autiosalo [24] apresenta um estudo sobre o uso de gêmeos digitais aplicados à indústria, no qual a plataforma proposta é usada para fins de pesquisa e ensino, assim como a nossa. A visualização dos dados do sensor é feita com óculos de realidade aumentada. Outro trabalho de aplicação na indústria apresenta o conceito de prova do desenvolvimento do sistema digital de criação de gêmeos [27], em que os autores usaram uma ferramenta CAD para visualizar os dados.

Outro sistema apresentado por Schroeder et al. [26] é focado apenas na especificação do modelo de dados, pois utiliza a plataforma FIWARE [23] como base para a camada digital. Em nossa proposta, escolhemos criar nossa própria camada digital, conforme será discutido posteriormente.

O trabalho de Um, Popper e Ruskowski [28] apresenta uma plataforma modular para criação de visualizações com realidade aumentada, bem como nossa proposta. Eles conduzem um estudo de caso aplicado à visualização digital de gêmeos em uma plataforma industrial. Em outro artigo, Schroeder et al. [29] discutem a criação de sistema digital de visualização dupla com RA. O sistema proposto é baseado no uso de serviços da Web e usa uma arquitetura cliente-servidor. Essa proposta de uso de serviços da web também foi seguida em nosso projeto. 
III.

O DESENVOLVIMENTO DO SISTEMA

Nosso sistema é composto de três camadas, conforme apresentado na Figura 1 (Física, Dados e Digital), no qual a Camada Física é composta pelos robôs, microcontroladores, sensores, dispositivos e conexões de transmissões físicas. Os sensores são conectados em microcontroladores montados aos robôs. Este é o cenário físico mínimo para suportar a Internet do ambiente de comunicação das coisas.

As Camadas de Dados, que representam os metadados trocados entre a Camada Física e a Camada Digital, são compostas de bancos de dados e protocolos de metadados. O protocolo de metadata usado em nosso sistema é o MQTT [33]. Quando os microcontroladores são inicializados, eles se conectam ao WIFI e ao Broker MQTT.

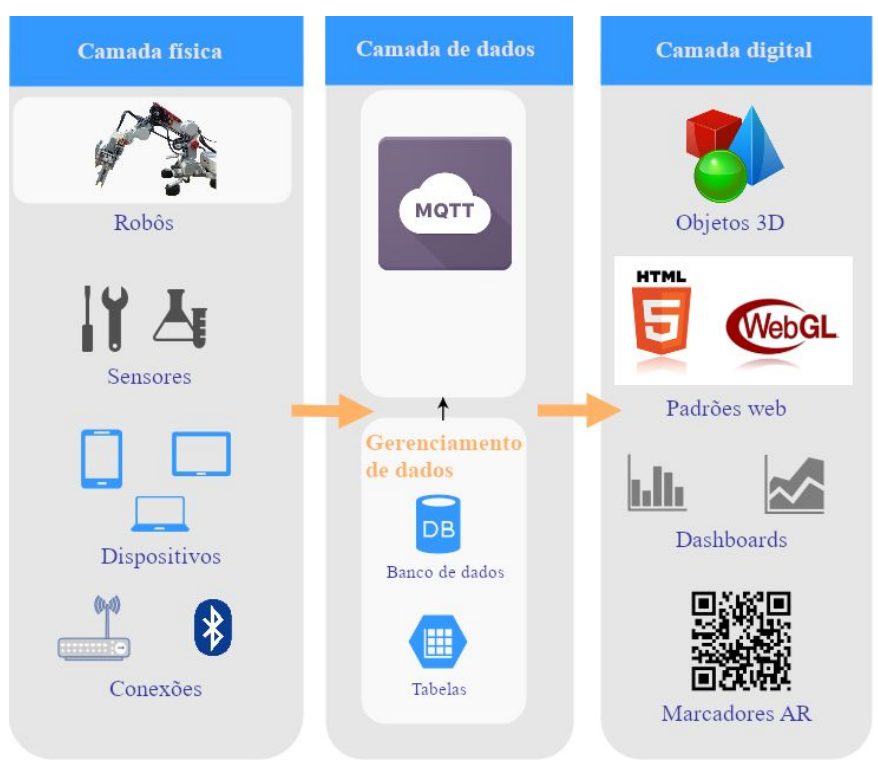

Fig. 1. Arquitetura do sistema em 3 camadas: Camada física (Esquerda), Camada de dados(Meio) e Camada Digital (Direita).

Através da Camada Digital é possível enviar comandos para o braço do robô, ombros, cotovelos ou efetores finais, esses comandos são enviados usando o protocolo MQTT para que múltiplos microcontroladores possam enviar e receber informações dinamicamente. A visualização da Camada Digital em 3D é criada com a biblioteca Three.js e com um braço robótico feito com blocos, como exemplo. A informação adquirida do sensor pode ser visualizada por cores no modelo. O Digital Layer também possui uma versão do painel com gráficos, criado com o serviço da web ThingSpeak. para permitir um histórico dos dados e uma visualização em tempo real. cuja visualização pode ser vista na Figura 2.

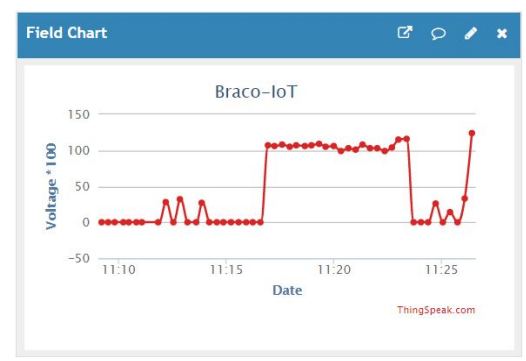


Fig. 2. Dashboard do sistema criado no ThingSpeak

\section{A. O fluxo de execução do sistema}

O sistema assimila os estados de seus componentes, portanto, se um comando for enviado para um motor, o sistema esperará uma resposta do microcontrolador informando se ele está ligado ou não. A resposta é o sinal de dois codificadores rotativos embutidos nos motores do robô, que enviam dois valores para os microcontroladores, um 0 ou 1 . A combinação de 0 s e 1 s diz se o motor está funcionando para frente, para trás ou se não está se movendo. Então, com esse feedback o microcontrolador envia uma string para a camada de dados informando qual direção o motor está executando e move o modelo 3D de acordo. Na Figura 4 é ilustrada a visualização 3D dessa camada digital.

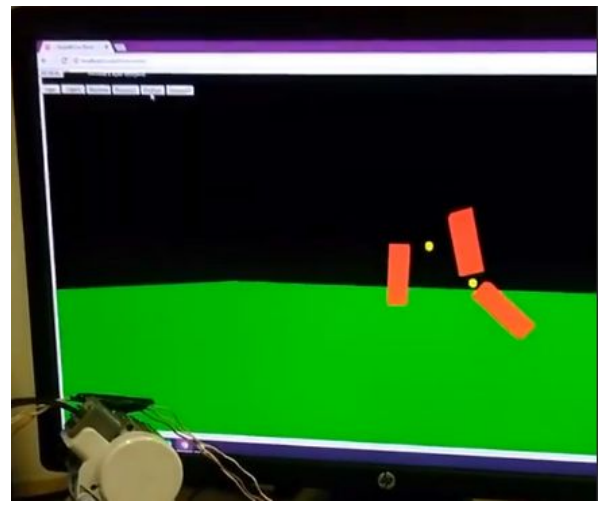

Fig. 3. A visualização da simulação 3D

O medidor de tensão, construído com dois resistores $1 \mathrm{k}$ Ohms para manter a tensão abaixo de 3,3, envia para a camada de dados um valor que corresponde a qual tensão os motores estão usando. Se o uso da tensão cai, significa que o motor está sob carga, mostrado na simulação 3D. O método usado para medição de carga é baseado na produção de CEMF (Força Contra-Eletromotriz) durante o movimento do motor. A corrente do sistema é calculada pela diferença entre a tensão aplicada e a CEMF, portanto, se a rotação for alta, a tensão final é menor que a tensão aplicada e a corrente diminui; se a carga é aplicada, a rotação é baixa, a tensão final é próximo ao valor de tensão aplicado e a corrente aumenta e causa uma queda de tensão na resistência interna da fonte de alimentação.

$$
V_{S}-V_{C E M F}=I . R(1)
$$

Em que Vs é o suprimento de tensão, Vcemf é o CEMF produzido, I é a corrente e R é a resistência. 


\section{B. Outras interfaces do sistema}

O sistema também possui uma interface para exibir dados com RA baseado em marcadores fiduciais [34], devido ao seu baixo custo e robustez. Este serviço de realidade aumentada foi criado com o mecanismo de jogos Unity [35] e com a biblioteca Vuforia [36].

\section{Resultados}

\section{A. Materiais e métodos}

Para validar nosso sistema, desenvolvemos uma Camada Física com um braço robótico e seu Gêmeo Digital. O braço do robô foi construído com LEGO porque é de fácil montagem e desmontagem, possui escalabilidade e também dá a possibilidade de criar um kit educacional sobre o Industry 4.0. O robô é composto de poucas partes, quatro motores NXT, dois como o ombro, um como o cotovelo, e o efetor final, feixes e engrenagens LEGO Technic para redução entre os motores, como ilustrado na Figura 4.

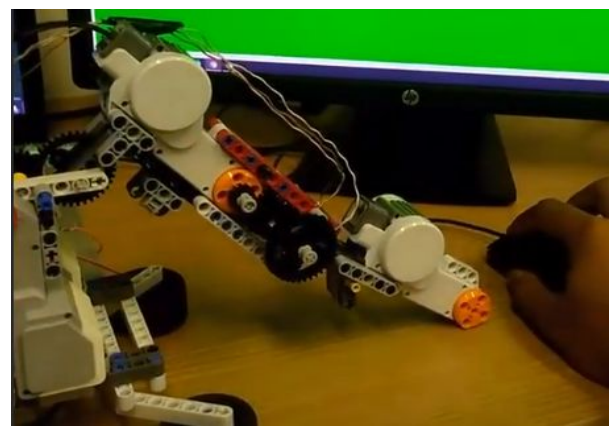

Fig. 4. Um braço robótico feito em Lego para validar o sistema

O circuito dos sensores foi composto por dois NODEMCU ESP8266, dois shields de motor para o NODEMCU, codificadores rotativos (incorporados aos motores), botões como end-stop, um PCF 8591 para permitir a conversão analógica para digital e divisores de tensão como sensores para leitura da voltagem dos motores.

\section{B. Experimentos}

Para validar o sistema, foi realizado um experimento em que uma carga é aplicada ao braço físico. Ao receber essa carga, os sensores do braço devem enviar um sinal para que ela seja armazenada na Camada de Dados, que, por sua vez, deve enviar uma mensagem para a camada digital para que a carga seja representada no ambiente de 
simulação. O resultado visual na simulação deve ser uma mudança na cor do eixo afetado pela carga, ilustrado na Figura 5.

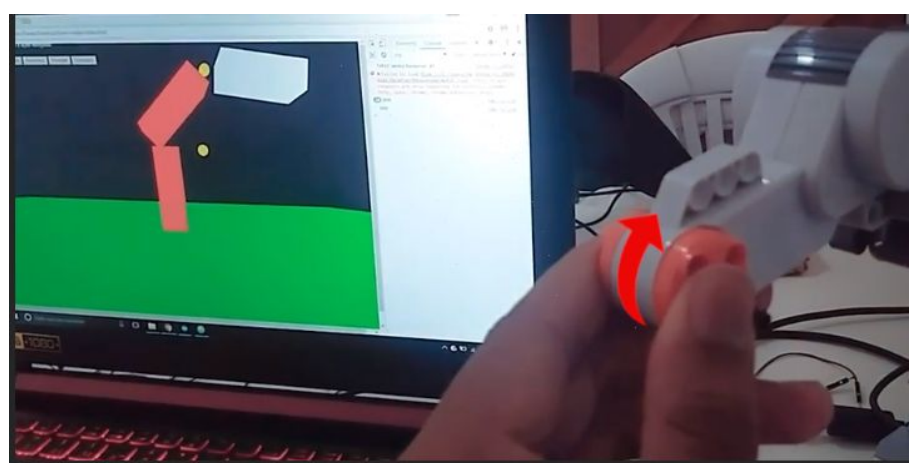

Fig. 5. Teste da validação do Gêmeo Digital. Quando o motor tem carga o Gêmeo Digital se altera. $O$ robô digital, inicialmente é todo vermelho, mas quando é aplicada carga, um módulo muda de cor. A seta vermelha indica a direção da rotação do motor.

Em outro experimento, foi realizado um teste com o módulo de realidade aumentada. Neste teste, uma aplicação móvel reconhece um marcador fiducial adequado para RA. Ao reconhecer o marcador, o aplicativo desenha uma interface com botões de controle, que permitem ligar desligue o braço ou mudar a direção da sua rotação. A interface ainda exibe um quadrado rotativo que ilustra a direção atual da rotação. $O$ ambiente do segundo experimento pode ser visto na Figura 6, na qual observa-se que,o aplicativo está sendo executado em um dispositivo móvel. Neste caso, é o dispositivo móvel que envia os sinais para a camada de dados, o que demonstra que nossa arquitetura suporta diferentes dispositivos integrados ao sistema, em que todos esses dispositivos podem enviar sinais de controle para a camada de dados.

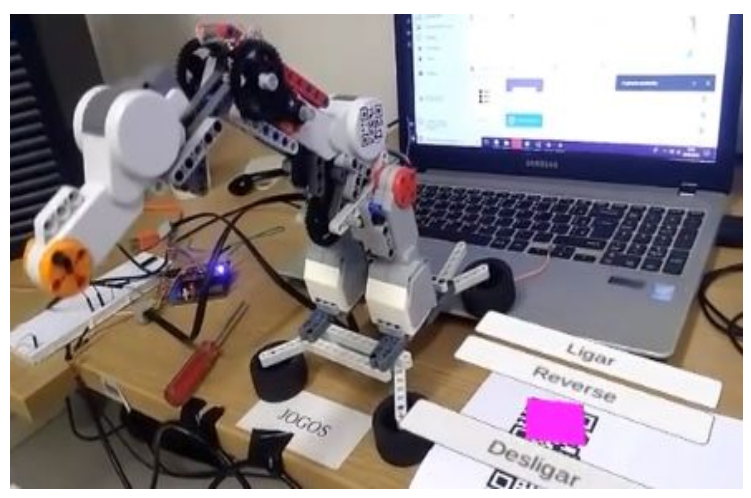

Fig. 6. Aplicação de Realidade Aumentada para controlar o braço robótico.

O segundo experimento também demonstra que o dispositivo móvel é capaz de receber mensagens da camada de dados. Quando a interface é desenhada na tela do 
dispositivo, ela recebe a direção atual da rotação do braço. Esta informação é representada graficamente na interface. Sempre que a seta de rotação muda, sua representação gráfica é alterada na interface do dispositivo.

\section{Conclusão}

A ideia de mesclar todos esses conceitos permite uma coleta de dados constante e dinâmica, ou seja, o sistema pode ler o estado de seus elementos e assimilar os resultados várias vezes por segundo. Para exemplificar, basta imaginar que um braço robótico é responsável por levantar um chassi de 1,5 toneladas, ao analisar a corrente consumida em cada motor, podemos conhecer os dados sobre a tarefa que o braço executa. A partir disso com base na média destes os valores calculados o sistema pode identificar que o equipamento está gasto e precisa de manutenção, e então o Gêmeo Virtual dá indicações visuais dos problemas, além de mostrar os dados em si, ele altera a cor dos elementos no ambiente virtual.

Esse modelo fornece uma base de controle para todo o processo de produção de cada elemento da linha e de cada componente dos elementos, integrando tudo isso em uma simulação virtual, criando, assim, uma plataforma mais amigável para a análise de dados, o que significa custo e o tempo de manutenção de equipamentos reduzidos a margens vantajosas para indústrias.

\section{REFERENCES}

[1] Datta, Shoumen Palit Austin. "Emergence of Digital Twins-Is this the march of reason?." Journal of Innovation Management 5, no. 3 (2017): 14-33.

[2] Kopetz, Hermann. "Internet of things." In Real-time systems, pp. 307-323. Springer, Boston, MA, 2011.

[3] Hirmer, Pascal, Uwe Breitenbücher, Ana Cristina Franco da Silva, Kálmán Képes, Bernhard Mitschang, and Matthias Wieland. "Automating the Provisioning and Configuration of Devices in the Internet of Things." CSIMQ 9 (2016): 28-43.

[4] Grieves, Michael W. "Virtually Indistinguishable." In IFIP International Conference on Product Lifecycle Management, pp. 226-242. Springer, Berlin, Heidelberg, 2012.

[5] Grieves, Michael. "Product Lifecycle Development." (2005).

[6] Grieves, Michael. "Virtually Perfect." (2011).

[7] Tao, Fei, Jiangfeng Cheng, Qinglin Qi, Meng Zhang, He Zhang, and Fangyuan Sui. "Digital twin-driven product design, manufacturing and service with big data." The International Journal of Advanced Manufacturing Technology 94, no. 9-12 (2018): 3563-3576.

[8] Glaessgen, Edward, and David Stargel. "The digital twin paradigm for future NASA and US Air Force vehicles." In 53rd AIAA/ASME/ASCE/AHS/ASC Structures, Structural Dynamics and Materials Conference 20th AIAA/ASME/AHS Adaptive Structures Conference 14th AIAA, p. 1818. 2012. 
[9] Tuegel, Eric J., Anthony R. Ingraffea, Thomas G. Eason, and S. Michael Spottswood. "Reengineering aircraft structural life prediction using a digital twin." International Journal of Aerospace Engineering 2011 (2011).

[10] Greenwood, J. (1997). The third industrial revolution: technology, productivity, and income inequality (No. 435). American Enterprise Institute.

[11] Kortuem, G., Kawsar, F., Sundramoorthy, V., \& Fitton, D. (2010). Smart objects as building blocks for the internet of things. IEEE Internet Computing, 14(1), 44-51.

[12] Xia, F., Yang, L. T., Wang, L., \& Vinel, A. (2012). Internet of things. International Journal of Communication Systems, 25(9), 1101.

[13] Lasi, H., Fettke, P., Kemper, H. G., Feld, T., \& Hoffmann, M. (2014). Industry 4.0. Business \& Information Systems Engineering, 6(4), 239-242.

[14] Weyer, S., Schmitt, M., Ohmer, M., \& Gorecky, D. (2015). Towards Industry 4.0-Standardization as the crucial challenge for highly modular, multi-vendor production systems. Ifac-Papersonline, 48(3), 579-584.

[15] Baheti, R., \& Gill, H. (2011). Cyber-physical systems. The impact of control technology, 12, 161-166.

[16] Gajski, D. D., Vahid, F., Narayan, S., \& Gong, J. (1994). Specification and design of embedded systems.

[17] Wolf, W. (2009). Cyber-physical systems. Computer, 42(3), 88-89.

[18] Neidig, J., \& Stephan, P. (2009, October). An Object Memory Modeling Approach for Product Life Cycle Applications. In Intelligent Environments (Workshops) (pp. 27-32).

[19] Gorecky, D., Schmitt, M., Loskyll, M., \& Zühlke, D. (2014, July). Human-machine-interaction in the industry 4.0 era. In Industrial Informatics (INDIN), 2014 12th IEEE International Conference on (pp. 289-294). IEEE.

[20] Azuma, R. T. (1997). A survey of augmented reality. Presence: Teleoperators \& Virtual Environments, 6(4), 355-385.

[21] Burdea, G. C., \& Coiffet, P. (2003). Virtual reality technology (Vol. 1). John Wiley \& Sons.

[22] Verdouw, C.N. and Kruize, J.W., Digital twins in farm management: illustrations from the FIWARE accelerators SmartAgriFood and Fractals.

[23] Stravoskoufos, K., Sotiriadis, S. and Petrakis, E., 2016, April. IoT-A and FIWARE: bridging the barriers between the Cloud and IoT systems design and implementation. In Proc. 6th Int'l Conf. Cloud Computing and Services Science (pp. 146-153).

[24] Autiosalo, J., 2018, February. Platform for industrial internet and digital twin focused education, research, and innovation: Ilmatar the overhead crane. In Internet of Things (WF-IoT), 2018 IEEE 4th World Forum on (pp. 241-244). IEEE.

[25] Luo, W., Hu, T., Zhu, W. and Tao, F., 2018, March. Digital twin modeling method for CNC machine tool. In Networking, Sensing and Control (ICNSC), 2018 IEEE 15th International Conference on (pp. 1-4). IEEE. 
[26] Schroeder, G.N., Steinmetz, C., Pereira, C.E. and Espindola, D.B., 2016. Digital twin data modeling with automationML and a communication methodology for data exchange. IFAC-Papers OnLine, 49(30), pp.12-17.

[27] Haag, S. and Anderl, R., 2018. Digital twin-Proof of concept. Manufacturing Letters, 15, pp.64-66.

[28] Um, J., Popper, J. and Ruskowski, M., 2018, May. Modular augmented reality platform for smart operator in production environment. In 2018 IEEE Industrial Cyber-Physical Systems (ICPS) (pp. 720-725). IEEE.

[29] Schroeder, G., Steinmetz, C., Pereira, C.E., Muller, I., Garcia, N., Espindola, D. and Rodrigues, R., 2016, July. Visualising the digital twin using web services and augmented reality. In Industrial Informatics (INDIN), 2016 IEEE 14th International Conference on (pp. 522-527). IEEE.

[30] Dawkins, O., Dennett, A. and Hudson-Smith, A., (2018). Living with a Digital Twin: Operational management and engagement using IoT and Mixed Realities at UCL's Here East Campus on the Queen Elizabeth Olympic Park.

[31] Damiani, L., Demartini, M., Giribone, P., Maggiani, M., Revetria, R. and Tonelli, F., (2018). Simulation and Digital Twin Based Design of a Production Line: A Case Study. In Proceedings of the International MultiConference of Engineers and Computer Scientists (Vol. 2).

[32] Hirmer, P., Breitenbücher, U., da Silva, A.C.F., Képes, K., Mitschang, B. and Wieland, M., (2016). Automating the Provisioning and Configuration of Devices in the Internet of Things. CSIMQ, 9, pp.28-43.

[33] Luzuriaga, J.E., Cano, J.C., Calafate, C., Manzoni, P., Perez, M. and Boronat, P., (2015), September. Handling mobility in IoT applications using the MQTT protocol. In Internet Technologies and Applications (ITA), 2015 (pp. 245-250). IEEE.

[34] Van Krevelen, D.W.F. and Poelman, R., (2010). A survey of augmented reality technologies, applications and limitations. International journal of virtual reality, 9(2), p.1.

[35] Xie, J., (2012), July. Research on key technologies base Unity3D game engine. In Computer Science \& Education (ICCSE), 2012 7th International Conference on (pp. 695-699). IEEE.

[36] Amin, D. and Govilkar, S., (2015). Comparative study of augmented reality SDKs. International Journal on Computational Science \& Applications, 5(1), pp.11-26. 